\title{
Das Verhältnis von Nichtregierungsorganisationen und privaten Unternehmen in der globalen Umwelt-, Menschenrechts- und Sicherheitspolitik
}

\author{
Thomas Hickmann • Berenike Prem
}

Angenommen: 7. Juni 2021 / Online publiziert: 13. Juli 2021

(C) Der/die Autor(en) 2021

Zusammenfassung In der Literatur herrscht weitgehend Einigkeit, dass sich das Verhältnis von Nichtregierungsorganisationen (NGOs) und privaten Unternehmen in den vergangenen Jahren erweitert hat. Dennoch sind vergleichende Studien zur Interaktion zwischen beiden Akteursgruppen über verschiedene Politikfelder hinweg bislang selten. Gemeinsame Strukturen und Trends bleiben somit unterbeleuchtet. Der vorliegende Aufsatz untersucht daher das Verhältnis von NGOs und dem Unternehmenssektor in der globalen Umwelt-, Menschenrechts- und Sicherheitspolitik. Die Analyse zeigt, dass einige Entwicklungen politikfeldspezifisch sind. So fällt der Protest von NGOs gegenüber der privaten Sicherheitsbranche relativ zahm aus. Zugleich beobachten wir einen politikfeldübergreifenden Trend, der sich in einer graduellen Funktionserweiterung von NGOs im Bemühen um normkonformes Unternehmenshandeln äußert. NGOs agieren dabei zunehmend als Ko-Regulierer, indem sie mit Unternehmensvertreter*innen in Multi-Stakeholder-Initiativen gemeinsame Regeln erarbeiten, deren Einhaltung überwachen und für deren Umsetzung sorgen. Unsere Analyse mündet in der These, dass dieser Wandel durch die Diffusion eines globalen Skripts begriffen werden kann, das die Einbindung privatwirtschaftlicher Akteure zur Lösung globaler Probleme vorsieht.

Schlüsselwörter Menschenrechtspolitik · Nichtregierungsorganisationen · Private Unternehmen · Umweltpolitik · Sicherheitspolitik · Globales Skript

\footnotetext{
Thomas Hickmann $(\square)$

Copernicus Institute of Sustainable Development, Universiteit Utrecht, Utrecht, Niederlande

E-Mail: t.hickmann@uu.nl

Berenike Prem

Institut für Internationale und Interkulturelle Studien (InIIS), Universität Bremen, Bremen, Deutschland

E-Mail: berenike.prem@uni-bremen.de
} 


\title{
The relationship between nongovernmental organizations and private corporations in global environmental, human rights and security politics
}

\begin{abstract}
There is a widespread consensus in the literature that the relationship between non-governmental organizations (NGOs) and private corporations has broadened over time. And yet there is a dearth of comparative studies on the interaction between both types of actors across different policy fields. Common structures and trends thus remain underexposed. To address this gap, the present article examines the relationship between NGOs and the corporate sector in global environmental, human rights and security politics. The analysis reveals some policy field-specific features in the relationship between NGOs and business. For instance, NGO campaigns that target the private security industry turn out relatively tame. At the same time, we find support for an emerging pattern across policy fields insofar as NGOs have gradually broadened their repertoire of action in their attempt to push firms to meet social norms. NGOs increasingly act as co-regulators by joining forces with business representatives in multi-stakeholder initiatives that set rules for business conduct, monitor compliance and help firms implement their commitments. We suggest that this transformation can be captured by the diffusion of a global script that spells out an expectation to include private actors into solving global challenges.
\end{abstract}

Schlüsselwörter Human rights · Nongovernmental organizations · Business · Environmental policy $\cdot$ Security policy $\cdot$ Global script

\section{Einleitung}

In der politikwissenschaftlichen Literatur werden Nichtregierungsorganisationen (NGOs) klassischerweise als Gegenspielerinnen privater Unternehmen behandelt. ${ }^{1}$ An ihre Rolle in der Weltpolitik ist häufig die Hoffnung geknüpft, die Macht privatwirtschaftlicher Akteure zu begrenzen und einzudämmen. Als ein gegenläufiger Trend gilt die Entwicklung kooperativer Formen der Zusammenarbeit in Selbstregulierungs- und sogenannten Multi-Stakeholder-Initiativen (MSIs) (BaumannPauly et al. 2016; Witte et al. 2000). Die globale Klimapolitik gilt als exemplarisch für die Entstehung dieses neuen Partnerschafts-Paradigmas (Hickmann und Prem 2018). Auch in anderen Politikfeldern lässt sich eine ähnliche Entwicklung erkennen. Allerdings gibt es bislang keinen systematischen Vergleich, um die Reichweite dieses Trends zu belegen und gemeinsame Strukturen und Trends bleiben somit unterbeleuchtet. Die meisten Studien widmen sich dem Funktionswandel von NGOs gegenüber der Privatwirtschaft in einzelnen Politikbereichen und legen dabei sehr unterschiedliche Maßstäbe an. Handelt es sich also bei der zunehmenden

\footnotetext{
1 Unter den Begriff der NGOs fassen wir in diesem Aufsatz in Abgrenzung zum Privatsektor nur solche Akteure, deren Tätigkeiten nicht auf die Maximierung des eigenen Gewinns gerichtet sind, sondern die sich in erster Linie für die Steigerung des Gemeinwohls einsetzen.
} 
Zusammenarbeit beider Akteursgruppen um ein begrenztes, politikfeldspezifisches Phänomen oder um einen generellen Trend?

Um diese Frage beantworten zu können, untersucht der vorliegende Aufsatz die Interaktionen zwischen NGOs und der Privatwirtschaft in der globalen Umwelt-, Menschenrechts- und Sicherheitspolitik. In der Forschung wird häufig zwischen konfrontativen und kooperativen Strategien unterschieden, auf die NGOs in ihrem Ringen um verantwortliches Unternehmenshandeln zurückgreifen (Yaziji und Doh 2009; van Huijstee und Glasbergen 2010; Coni-Zimmer und Flohr 2015). Diese Kategorisierung birgt jedoch eine gewisse Unschärfe. Denn sie übersieht, dass NGOs zur Durchsetzung ihrer Anliegen ein sehr viel breiteres Handlungsspektrum ausschöpfen. Darüber hinaus verleitet eine solche Betrachtung dazu, die Qualität zivilgesellschaftlicher Aktivität als genuinen Beitrag zur Regulierung privater Unternehmen zu unterschätzen. In einer solchen Perspektive steht die Konflikthaftigkeit bzw. die Kooperationsbeziehung beider Akteure im Vordergrund und weniger die Frage, welche Funktionen NGOs in der Weltpolitik ausüben.

Zur Analyse der NGO-Funktionen, die auf Unternehmen gerichtet sind, macht unser Beitrag aus diesem Grund Anleihen aus der Governance-Forschung. Wir gruppieren die verschiedenen Aktivitäten von NGOs in die jeweiligen Phasen des Politikformulierungsprozesses ein, beginnend beim Agenda-Setting über Aufklärung, Regelsetzung, Implementierung bis zur Kontrolle der Regeleinhaltung. Zunächst ließe sich die Vermutung aufstellen, dass sich bei einem politikfeldübergreifenden Vergleich ein uneinheitliches Bild ergibt. So argumentieren verschiedene Autoren, dass die Formalisierung der Beziehung von NGOs zum Unternehmenssektor abnimmt, je härter die Politikfelder sind, also je näher sie an den hard issues der globalen Sicherheitspolitik reichen (e.g. Zangl und Zürn 2003, S. 172-176).

Zwar deutet unsere Analyse auf Unterschiede zwischen den Politikfeldern hin so fällt der Protest von NGOs gegen die private Sicherheitsbranche im Vergleich zur Umwelt- und Menschenrechtspolitik überraschend zahm aus. Dennoch beobachten wir einen klaren Trend über die untersuchten Politikfelder hinweg: Neben ihrer Rolle als Agenda-Setter und Informationsvermittler betätigen sich NGOs in allen drei Feldern auch als Ko-Regulierer, indem sie mit Unternehmensvertretern in MSIs gemeinsame Regeln erarbeiten, deren Einhaltung überwachen und (innerhalb oder außerhalb von MSIs) für deren Umsetzung sorgen. In Anlehnung an den soziologischen Institutionalismus argumentieren wir, dass dieser Wandel durch die Diffusion eines globalen Skripts erklärt werden kann, das die Einbindung privatwirtschaftlicher Akteure zur Lösung globaler Probleme vorsieht.

Unser Beitrag gliedert sich in fünf Abschnitte. Im Anschluss an diese Einleitung formulieren wir zunächst unseren Analyserahmen und diskutieren mit Bezug zur Governance-Forschung unterschiedliche NGO-Aktivitäten im Politikformulierungsprozess. Auf Basis dieses konzeptionellen Rahmens untersuchen wir im empirischen Teil die Zusammenarbeit von NGOs und privaten Unternehmen in der globalen Umwelt-, Menschenrechts- und Sicherheitspolitik. Im Fazit fassen wir unsere Ergebnisse aus der Analyse der drei Politikfelder zusammen und zeigen einige weiterführende Forschungsperspektiven auf. 


\section{Die Rolle von NGOs im Politikformulierungsprozess}

In den Internationalen Beziehungen (IB) sind sowohl NGOs als auch private Unternehmen verhältnismäßig junge Forschungsgegenstände. Dabei lag das Interesse lange auf dem Einfluss dieser Akteure auf zwischenstaatliche Entscheidungsprozesse (Klotz 1995; Risse-Kappen 1995; Keck und Sikkink 1998). Erst mit der Governance-Wende in den IB wurde NGOs und privaten Unternehmen ein eigenständiger Akteurstatus zugeschrieben (e.g. Cutler et al. 1999; Hall und Biersteker 2002; Avant et al. 2010). In diesem Zusammenhang wurden ebenfalls die Interaktionen zwischen NGOs und dem Privatsektor stärker in den Blick genommen.

Dieses Verhältnis scheint sich in den vergangenen Jahren Stück für Stück erweitert zu haben (Coni-Zimmer und Flohr 2015; Yaziji und Doh 2009). NGOs treten nicht nur als Protestbewegungen auf, sondern arbeiten zunehmend mit dem Privatsektor zusammen, um Unternehmenspraktiken zu verändern. In ihrem Bemühen um verantwortliches und normkonformes Unternehmenshandeln greifen NGOs auf ein breites Handlungsrepertoire zurück, das wir in Anlehnung an die Governance-Forschung den unterschiedlichen Phasen des Policy Cycles zuordnen: Agenda-Setting, Aufklärung, Regelsetzung, Implementierung und Regelüberwachung (e.g. Abbott und Snidal 2009; Eberlein et al. 2014). Auch wenn diese idealtypischen NGO-Funktionen nicht immer klar voneinander abzugrenzen sind, unterscheiden sie sich durch ihren steigenden Grad der Institutionalisierung. In der Bewegungsforschung bezeichnet der Prozess der Institutionalisierung die zunehmende Formalisierung der Beziehungen zivilgesellschaftlicher Akteure. Dieser Prozess geht oft mit ihrer Einbettung und Integration in formale Politikprozesse einher: ,,movements can be institutionalized in the sense that they operate within social institutions and organizations" (Staggenborg 2013).

1. Agenda-Setting: Erstens schaffen NGOs ein öffentliches Problembewusstsein für bestimmte Unternehmenspraktiken und setzen ihre Anliegen auf die unternehmenspolitische und/oder öffentliche Agenda (Keck und Sikkink 1998; Price 2003; Carpenter 2007a). Sie nehmen damit eine Funktion als Watchdogs wahr, die das Verhalten von transnationalen Unternehmen an bestimmten Normen und Wertevorstellungen messen. Bei wahrgenommenen Normverstößen schlagen sie Alarm und greifen auf öffentliches Naming and Shaming zurück (Utting 2005, S. 377; Curbach 2008, S. 375). Das wichtigste Medium, mit dem NGOs auf Missstände im Verhalten eines Unternehmens (und auf vorhandene oder wahrgenommene Regelungslücken) aufmerksam machen können, sind transnationale Kampagnen, die zum Kerngeschäft unternehmenskritischer NGOs gehören (Yaziji und Doh 2009, S. 60). Ziel solcher Kampagnen ist es, Druck auf transnationale Unternehmen auszuüben, um sie zu Änderungen ihres Verhaltens zu bewegen. Der Erfolg solcher Kampagnen hängt im Wesentlichen von der Fähigkeit ab, Themen unter Bezugnahme auf allgemein akzeptierte Normen zu beeinflussen (Framing) (Shawki 2010).

2. Aufklärung: NGOs liefern nicht nur Einblicke in Missstände unternehmerischer Praktiken vor Ort, sondern vermitteln auch theoretisches und technisches Wissen, das in die Definition von Problemen und Politiken einfließt (Utting 2005, S. 377; 
Boström und Hallström 2010, S. 45-46; Steffek 2013, S. 1007). In diesem Sinne nehmen NGOs eine wichtige Expert*innenfunktion ein. Die Verbreitung von Informationen zur Bewertung von anthropogen verursachten Umweltproblemen steht beispielhaft für diese Art der Aktivität, wie Betsill und Corell insbesondere für die internationale Klimapolitik zeigen (Betsill und Corell 2008). Dabei fertigen NGOs zum Beispiel zusammenfassende Berichte der komplexen Verhandlungsgegenstände an, stellen Informationen für die Verhandlungsparteien bereit und beteiligen sich an Diskussionen mit Entscheidungsträger*innen (Yamin 2001). Ebenso liefern NGOs Berichte und Datenmaterial an Unternehmen, um die Wirkung der Unternehmenspraktiken auf die Umwelt und Gesellschaft zu belegen. In dieser Phase ist der direkte Zugang zu Verhandlungen und/oder Unternehmen eine wichtige Voraussetzung für NGOs, um sich Gehör zu verschaffen.

3. Regelsetzung: Drittens treten NGOs als Partner bei der Formulierung von Verhaltenskodizes und Zertifizierungsstandards für Unternehmenspraktiken auf. Die häufigste Form dieser Funktion von NGOs ist die Beteiligung an Regelsetzungsprozessen auf konsultativer Basis (Börzel und Risse 2005, S. 199). Staaten und/ oder internationale Organisationen bleiben hier meist die treibende Kraft hinter den auszuhandelnden Richtlinien und bieten nichtstaatlichen Akteuren eine lose Interaktionsplattform. Davon unterscheiden sich Formen der Ko-Regulierung, in denen NGOs als gleichberechtigte Partnerinnen auftreten (Tosun et al. 2016). MSIs wie die Fair Labour Association (Textilsektor) sind Beispiele für solche ko-regulativen Arrangements. Sie bestehen aus freiwilligen Zusammenschlüssen zivilgesellschaftlicher, privater und öffentlicher Akteure, deren Ziel es ist, gesellschaftliche Probleme in kooperativer Weise zu lösen (Andonova 2010).

4. Implementierung: Viertens betätigen sich NGOs als Umsetzungsgehilfen, um die Einhaltung und Umsetzung bestimmter Standards und Normen zu gewährleisten. Dafür stellen sie ihre Expertise und praktische Ausführungshilfe für das freiwillige Engagement von Unternehmen bereit und nehmen dadurch direkt Einfluss auf unternehmerisches Handeln (Utting 2005, S. 378). Ein Beispiel hierfür ist die gemeinsame Umsetzung von Umweltschutzprojekten in Entwicklungs- und Schwellenländern durch den World Wide Fund for Nature oder andere eher wirtschaftsnahe NGOs, bei denen erneuerbare Energien und andere umweltschonende Technologien eingeführt werden.

5. Kontrolle der Regeleinhaltung: Schließlich nehmen NGOs eine Kontrollfunktion ein, indem sie die Einhaltung von Umwelt- und Sozialstandards überwachen. Dies kann einerseits über ihre Beteiligung an transnationalen Governance-Initiativen geschehen, insofern diese über institutionalisierte Berichterstattungspflichten oder Zertifizierungssysteme verfügen (Pattberg 2007; Gulbrandsen 2010). Andererseits kann diese Kontrollfunktion auch ein klassisches Dienstleistungsverhältnis annehmen, wie etwa im Fall der Rainforest Alliance, die für den ChiquitaKonzern bestimmte Monitoring-Leistungen erbringt und dessen Bananenplantagen auf die Einhaltung von Nachhaltigkeitsstandards überprüft sowie Zertifikate ausstellt (Bendell 2000).

Da diese Kategorien nicht immer trennscharf sind, fassen wir einzelne NGOFunktionen für die empirische Analyse zusammen. So fallen in den von uns ana- 
lysierten Fällen Regelsetzung, Überwachung und Implementierung durch die Mitwirkung von NGOs in MSIs vielfach zusammen. In diesen Fällen sprechen wir von Ko-Regulierung durch NGOs (Börzel und Risse 2007, S. 202). Auf Basis der verschiedenen Funktionen von NGOs im Politikformulierungsprozess überprüfen wir in den folgenden Abschnitten das Verhältnis zwischen NGOs und privaten Unternehmen in der Umwelt-, Menschenrechts- und Sicherheitspolitik. Unsere Auswahl der Fallbeispiele erhebt dabei nicht den Anspruch, einen vollständigen Überblick über die Interaktionen von NGOs und Unternehmen in den jeweiligen Politikfeldern zu liefern. Vielmehr handelt es sich bei den von uns gewählten Beispielen um prototypische Fälle der jeweiligen NGO-Funktionen im Umgang mit der Privatwirtschaft, die als Blaupause für die Generierung unserer Hypothese einer politikfeldübergreifenden Norm zur partnerschaftlichen Zusammenarbeit zwischen den beiden Sektoren dienen.

Im Vordergrund unserer explorativen Vergleichsstudie steht also das Ziel, Hypothesen zum Verhältnis von NGOs und privaten Unternehmen zu genieren. Diese Herangehensweise erlaubt eine erste qualitative Annäherung an den empirischen Trend, dass NGOs sich verstärkt in einer ko-regulierenden und partnerschaftlich mit Unternehmen interagierenden Rolle wiederfinden. Diese Beobachtung soll perspektivisch in die weitere Theoriebildung zur Herausbildung eines globalen Skripts einfließen. Die angeführten Fallbeispiele basieren auf einer umfangreichen Analyse der Fachliteratur sowie Inhaltsanalysen von Websites und Grundsatzpapieren von NGOs.

\section{NGOs und Unternehmen in der globalen Umweltpolitik}

Agenda-Setting In den 1990er-Jahren haben NGOs in der Umweltpolitik vor allem als Advocacy-Netzwerke auf sich aufmerksam gemacht, die darauf abzielen, bestimmte Unternehmenspraktiken öffentlich anzuprangern (Conca 1995; Raustiala 1997; Keck und Sikkink 1998). Eine der bekanntesten NGO-Kampagnen in dieser Zeit war die Auseinandersetzung um die Brent Spar zwischen Greenpeace und Shell (Scherler 1996; Entine 2002). Die Brent Spar war ein schwimmender Öltank in der Nordsee, der seit Mitte der 1970er-Jahre in Gebrauch war. Nachdem im Jahr 1991 eine Pipeline verlegt wurde, die das Öl direkt von den Förderorten abpumpte, wurde die Brent Spar überflüssig und stillgelegt (Jordan 1998, S. 602-603). Im Tank verblieben $150 \mathrm{t}$ Ölschlamm, Schwermetalle sowie radioaktive und andere Industrieabfälle. Nach einer Expertenuntersuchung bekam Shell vom britischen Department of Trade and Industry die Erlaubnis, die Brent Spar vor der Westküste Schottlands $\mathrm{zu}$ versenken. Laut der Untersuchung war dies sowohl die günstigste als auch die mit den geringsten Umweltrisiken behaftete Entsorgungsvariante.

Greenpeace lehnte diese Entsorgung auf dem Meeresboden jedoch ab. Im April 1995 besetzte eine Gruppe von Greenpeace-Aktivisten die Brent Spar für drei Wochen, während andere Greenpeace-Mitarbeiter*innen gleichzeitig eine Medienkampagne gegen die Versenkung des Öltanks initiierten (Jordan 1998). Shell betonte in relativ technischen und nüchternen Statements immer wieder die geringen Umweltrisiken einer Versenkung und die Gefahr des Abschleppens an Land. Greenpeace 
hingegen präsentierte in der Kampagne mehrere, oftmals emotional aufgeladene Gegenargumente (Entine 2002). Anhand der Brent Spar, die als erste von etwa 400 ähnlichen Konstruktionen ausrangiert werden sollte, zeichnete Greenpeace das Bild einer gefährlichen Entwicklung, die das Meer bald in eine Müllhalde verwandeln könne. Mangels belastbarer wissenschaftlicher Erkenntnisse über die Auswirkungen einer solchen Tiefseeentsorgung für die Umwelt betonte Greenpeace, dass unter diesen Umständen die Entsorgung nicht ausgeführt werden dürfe. Durch die Besetzung und die Medienkampagne gelang es Greenpeace, das Thema in ganz Europa in die Medien zu befördern.

Der Aufruf zu einem Boykott von Shell führte in Deutschland zu enormen Umsatzeinbußen (Bennie 1998, S. 399). Die Regierungen von Deutschland, den Niederlanden, Belgien und Dänemark riefen Shell und Großbritannien gleichermaßen dazu auf, die Versenkung zu stoppen (Nash 1995). Im Juni 1995 verkündete Shell schließlich, die Brent Spar nicht zu versenken, sondern an Land zu schleppen. Drei Jahre später wurde beschlossen, die Reste des Tanks zum Bau einer Kaimauer in Norwegen zu verwenden. Die Kampagne um die Brent Spar hatte weitreichende Konsequenzen. Eine Entsorgung von Öltanks auf dem Meeresboden wurde in der Folgezeit politisch undenkbar (Bennie 1998, S. 397). Staaten verhandelten ein internationales Abkommen über ein Verbot von Abfallbeseitigung im Meer und die Ölindustrie bemühte sich um ein umweltfreundlicheres Image (Jordan 2001, S. 7).

Informationsvermittlung Der in transnationalen Kampgagen aufgebaute Druck auf Unternehmen förderte eine weitere Dynamik, die spätestens seit den 1990erJahren in der globalen Umweltpolitik zu beobachten ist: der Aufstieg sogenannter privater Governance-Initiativen für nachhaltiges und sozial verantwortliches Unternehmenshandeln (Clapp 1998; Falkner 2003). Diese Initiativen reichen von Maßnahmen auf individueller Firmenebene über sektorenweite Verhaltenskodizes bis hin zu globalen Umweltmanagementsystemen wie die ISO 14001-Serie (Potoski und Prakash 2005). Zugleich rückte mit der Rio-Konferenz 1992 das Vorhaben, die (umweltpolitischen) Aktivitäten multinationaler Unternehmen verbindlich auf internationaler Ebene zu regulieren - etwa im Rahmen der United Nations Commission on Trade and Development (UNCTAD) - in weite Ferne (Newell 2000, S. 120). Auf dieser Konferenz beschloss die internationale Staatengemeinschaft mit der Agenda 21 ein globales Aktionsprogramm, das verschiedene Leitlinien zur Förderung einer nachhaltigen Entwicklung für das 21. Jahrhundert enthält. Darin wurde sowohl die Bedeutung privater Selbstregulierungsinitiativen bekräftigt, um nachhaltige Fortschritte bei der Bekämpfung globaler Umwelt- und Entwicklungsprobleme zu erreichen, als auch der Beitrag sektorenübergreifender Zusammenarbeit zwischen staatlichen, privatwirtschaftlichen und zivilgesellschaftlichen Akteuren.

Die Proliferation freiwilliger Verhaltensstandards schuf eine erhöhte Nachfrage an umweltorientierter Beratung, die häufig von NGOs bedient wurde. Mühle spricht in diesem Zusammenhang von einem regelrechten ,global CSR [Corporate Social Responsibility] consulting market in the making“ (2010, S. 232). NGOs liefern Erkenntnisse darüber, wie in der täglichen Unternehmenspraxis Energie und Emissionen eingespart werden können und stellen Informationen bereit, auf deren Basis Unternehmen eigene Nachhaltigkeitsstrategien formulieren (Argenti 2004). 
Beispielhaft hierfür ist die strategisch-inhaltliche Zusammenarbeit zwischen dem World Wide Fund for Nature und privaten Unternehmen mit dem Ziel, deren ökologischen Fußabdruck zu verringern (World Wide Fund for Nature 2020).

Ko-Regulierung Mit ihrem Bekenntnis zur partnerschaftlichen Zusammenarbeit zwischen den Sektoren wurde die Agenda 21 wegweisend für eine weitere viel diskutierte Entwicklung in der globalen Umweltpolitik. In den letzten Jahrzehnten haben NGOs zahlreiche Initiativen gegründet, um Unternehmen darin zu unterstützen, freiwillige Schritte zu einer umweltfreundlichen Produktionsweise einzuleiten (Bäckstrand et al. 2010; Pattberg 2012; Hickmann 2017). Eine der ältesten derartigen Initiativen ist der Forest Stewardship Council (FSC). Als in den 1970erJahren die Sorge über Abholzung und Waldsterben wuchs, wurden vermehrt Rufe nach einem Zertifizierungssystem für den Forstsektor laut. Verhandlungen darüber im Rahmen von intergouvernementalen Institutionen schlugen jedoch fehl (Bell und Hindmoor 2012, S. 148-149). Daraufhin formten mehrere Umweltorganisationen eine Arbeitsgruppe, um ein privates Zertifizierungssystem zu erarbeiten. Dies sollte als zivilgesellschaftliche Alternative zu den erfolglosen Verhandlungen präsentiert werden und der FSC wurde 1993 ins Leben gerufen (Dingwerth 2008).

Ziel des FSC ist es, eine umweltfreundliche, sozialförderliche und ökonomisch tragfähige Bewirtschaftung der Wälder zu fördern. Der FSC ist eine MSI, die als Mitgliedschaftsorganisation gegründet wurde. Sie besteht aus Vertretern von Umweltverbänden, der Forstwirtschaft, sozialen Bewegungen, Organisationen indigener Völker, Waldzertifizierungsorganisationen, der kommunalen Forstwirtschaft, Einzelhändlern und Herstellern sowie einzelnen Waldbesitzern. Große Aufmerksamkeit hat die Governance-Struktur des FSC erhalten. In der regelmäßig stattfindenden Generalversammlung werden Entscheidungen gemeinsam von Umweltorganisationen und privaten Unternehmen getroffen (Pattberg 2007). Diese Generalversammlung, in der jedes Mitglied stimmberechtigt ist, unterteilt sich dabei in eine Umwelt-, Wirtschafts- und Sozialkammer, die jeweils ein Drittel des Stimmrechts halten. Jede der Kammern ist zusätzlich in eine Nord- und Südkammer unterteilt, so dass Akteure aus dem globalen Norden und dem globalen Süden jeweils 50\% der Stimmen besitzen.

Für den Schutz der Wälder hat der FSC zehn wirtschaftliche, soziale und ökologische Prinzipien entwickelt, denen der Holzabbau und die Weiterverarbeitung genügen müssen, um eine entsprechende Zertifizierung zu erhalten. Diese Prinzipien wurden in Form von 56 Kriterien weiter spezifiziert. Da diese Kriterien immer noch sehr allgemein sind, delegiert der FSC die konkrete Standardsetzung an nationale Initiativen. Dadurch ergibt sich eine Vielzahl anerkannter Regional- und Nationalstandards (Dingwerth 2008, S. 57). Der FSC galt lange als Erfolgsgeschichte der Zusammenarbeit zwischen NGOs und privaten Unternehmen (e.g. Gulbrandsen 2004). Mit Hilfe der Initiative konnte die Fläche an zertifiziertem Wald von 7 Mio. Hektar (1997) auf 117 Mio. Hektar (2010) gesteigert werden (Bell und Hindmoor 2012), wenngleich der Großteil davon im globalen Norden zu finden ist (Dingwerth 2008). Zugleich diente der FSC als Laboratorium für andere Governance-Arrangements und hat den Weg bereitet, das Multi-Stakeholder Modell in ein weithin 
anerkanntes (umweltpolitisches) Instrument in der Standardsetzung, der Zertifizierung und Akkreditierung zu etablieren.

Initiativen wie der Marine Stewardship Council (MSC) sind vom partnerschaftlichen Ansatz des FSC inspiriert und teilen wesentliche institutionelle Merkmale, wie das Vorhandensein eines Gremiums, in dem unterschiedliche Stakeholder repräsentiert sind und Verfahrensnormen wie Transparenz, Inklusivität und Rechenschaftspflichtigkeit gelten (Dingwerth und Pattberg 2009; Ponte 2014). Der MSC wurde zunächst als Stiftung einzelner NGOs und Unternehmen gegründet und hat sich später zu einer MSI mit zahlreichen Mitgliedern aus der Privatwirtschaft und der Zivilgesellschaft entwickelt (Kalfagianni und Pattberg 2013, S. 186-187). Er hat zum Ziel, eine gesicherte und nachhaltige Nutzung der weltweiten Fischbestände durch ein Zertifizierungssystem sicherzustellen (Boström und Hallström 2010, S. 41). Der Multi-Stakeholder Ansatz hat auch Eingang in den ISEAL Code of Good Practice gefunden, der allgemeingültige Leitfäden für die Entwicklung und Umsetzung von Umwelt- und Sozialstandards formuliert (Loconto und Fouilleux 2014).

Diese Entwicklung sollte jedoch nicht darüber hinwegtäuschen, dass viele NGOs nach wie vor skeptisch gegenüber privaten oder hybriden Governance-Initiativen sind (Clapp 2005). Diese Skepsis wird auch an der Kritik gegenüber dem FSC und anderen Zertifizierungssystemen sichtbar, die nach Ansicht von vielen NGOs unzureichend umgesetzt und kontrolliert werden und es bislang nicht vermochten, die rasante Abholzung in vielen Entwicklungs- und Schwellenländern - auch durch FSCzertifizierte Unternehmen - zu verhindern (Greenpeace 2021). Aus diesem Grund hat sich das Gründungsmitglied Greenpeace bereits 2018 aus dem FSC zurückgezogen (Greenpeace 2018). Stattdessen drängt Greenpeace auf rechtlich verbindliche Instrumente auf nationaler und internationaler Ebene, um die Aktivitäten von Unternehmen zu regulieren. In den letzten Jahren lässt sich tatsächlich eine stärkere Verrechtlichung in diesem Bereich beobachten. Die Europäische Union und eine Reihe Holz- und Rohstoff importierender Länder haben jüngst Lieferkettengesetze erlassen, die Unternehmen verpflichten, ihrer Sorgfaltspflicht nachzukommen. (Moser und Leipold 2021; Bartley 2014).

\section{NGOs und Unternehmen in der globalen Menschenrechtspolitik}

Agenda-Setting Spätestens seit Mitte der 1980er-Jahre spielen NGOs eine wichtige Rolle in der globalen Menschenrechtspolitik. Als Teile transnationaler Netzwerke prangern sie weltweit Verstöße gegen geltende Menschenrechtsstandards an und versuchen normverletzende Akteure durch Naming and Shaming-Kampagnen zu einer Abkehr von Menschenrechtsverletzungen zu bewegen (Keck und Sikkink 1998). Anfang der 1990er-Jahre entwickelte sich aus den Aktivitäten einzelner Menschenrechts-NGOs, wie beispielsweise Amnesty International, eine globale Bewegung, die sich gegen die Produktionsbedingungen multinationaler Unternehmen richtete. Eines der bekanntesten Beispiele für eine solche Protestbewegung ist die AntiSweatshop-Kampagne gegen Nike (Ruggie 2013, S. 3). Durch einen Bericht des Arbeitsaktivisten Jeff Ballinger, der 1992 im Harper's Magazine veröffentlicht wurde und die niedrigen Löhne und ausbeuterischen Arbeitskonditionen in den Betrieben 
von indonesischen Nike-Zulieferern thematisierte, geriet Nike erstmals in die Kritik (DeWinter-Schmitt 2007, S. 146).

Der Bericht legte nahe, dass die Zulieferer von Nike von allen für den Export produzierenden Firmen am wenigsten zahlten (Harrison und Scorse 2010, S. 249). Nachdem die indonesische Regierung, verärgert über die negative Berichterstattung, Ballinger des Landes verwiesen hatte, gründete er 1994 die NGO Press for Change. Diese Organisation konzentrierte sich auf die Mobilisierung gegen Nike und sollte die Aufmerksamkeit auf die Arbeitsbedingungen in Zulieferbetrieben lenken (DeWinter-Schmitt 2007, S. 145-146). Durch eine 1996 begonnene Kooperation mit der NGO Global Exchange, die über deutlich mehr Ressourcen verfügte, konnte die Kampagne auf nationaler Ebene in den USA fortgeführt werden. In der Folgezeit veröffentlichte etwa die New York Times zwischen Juni 1996 und Juni 1997 acht kritische Kolumnen über die Geschäftspraktiken von Nike (DeWinter-Schmitt 2007, S. 147). Global Exchange organisierte auch den ersten International Nike Day of Protest, bei dem Ende 1997 in vielen Bundesstaaten und zwölf Ländern vor NikeLäden gegen die Unternehmenspraxis demonstriert wurde (Carty 2001, S. 41-42).

Ein anderer wichtiger Akteur in der Allianz gegen Nike war die Studentenbewegung United Students Against Sweatshops. Sie führte ab Ende der 1990er-Jahre Informationskampagnen an Universitäten durch und forderte, dass Sport- und Kleidungsausstattung, die in Campusgeschäften verkauft wurden, nicht in Sweatshops produziert werden dürften. Dadurch geriet Nike in Gefahr, den lukrativen Universitätsmarkt zu verlieren (Ruggie 2013, S. 4). Ebenso wurde Nike anlässlich der Konferenz der World Trade Organization im Jahr 1999 zu einer prominenten Zielscheibe der Demonstranten im „Battle of Seattle“ (Carty 2001, S. 40). Die Proteste wirkten sich so stark auf die Nachfrage aus, dass Mitarbeiter entlassen werden mussten und das Management sich zu einer Umkehr in seiner bisherigen Unternehmenspolitik gezwungen sah. Nike hatte bis zu diesem Zeitpunkt den Standpunkt vertreten, nicht für die Zustände bei Zulieferern verantwortlich zu sein, da die Firmen nicht zu Nike gehörten und deshalb auch nicht unter seiner Kontrolle standen. Zwar hatte Nike schon im Jahr 1992 einen Code of Conduct verabschiedet, um internationale Arbeitsstandards und höhere Löhne einzuführen. Dieses Vorhaben wurde jedoch erst 1996 vollständig umgesetzt, während der Druck auf die Firma weiter stieg (Murphy und Mathew 2001, S. 6). Im Mai 1998 verkündete Nike schließlich die Kehrtwende und versprach weitreichende Änderungen in der Produktion. In den folgenden Jahren traf das Unternehmen Maßnahmen, die das Unternehmen zu einem Vorreiter im Bereich der gesellschaftlichen Verantwortung machten (Hayhurst und Szto 2016, S. 523).

Das in solchen NGO-Kampagnen geschaffene Problembewusstsein für Menschenrechtsverstöße durch Unternehmen hat auch im Rahmen des Menschenrechtsregimes der Vereinten Nationen (UN) zu einer Reihe von Initiativen geführt, die darauf abzielen, Normen, Regeln und Überprüfungsmechanismen für das Verhalten von Unternehmen zu etablieren. Einer der bedeutendsten Grundsätze sind die vom UN-Sonderbeauftragten für Menschenrechte und transnationaler Unternehmen, John Ruggie, entwickelten und 2011 durch den UN-Menschenrechtsrat verabschiedeten Leitprinzipen für Wirtschaft und Menschenrechte. Sie umfassen 1) die Verpflichtung von Staaten zum Schutz vor Menschenrechtsverletzungen durch Unternehmen; 2) die 
unternehmerische Verantwortung, Menschenrechte zu achten; sowie 3) den Zugang zu Abhilfe in Form von Rechtsmitteln und Wiedergutmachung. Für den Ruggie-Prozess selbst ist bezeichnend, dass er sich dem partnerschaftlichen Ansatz verpflichtet fühlte, der seit der Rio-Konferenz Einzug ins UN-System gefunden hat. Er war als bereiter Multi-Stakeholder-Konsultationsprozess angelegt, an dem sowohl Unternehmen als auch NGOs beteiligt waren. Die Einbindung unterschiedlicher Stakeholder und der erreichte Konsens aller beteiligten Akteure wird von den Befürworter*innen der Leitprinzipien als Argument für deren Legitimität hervorgehoben (Hamm 2016, S. 486).

Wegen ihrer rechtlichen Unverbindlichkeit sehen viele NGOs die Leitprinzipien jedoch kritisch (Albin-Lackey 2013). Befeuert durch Fälle schwerwiegender Verstöße gegen Menschenrechts- und Arbeitsschutzstandards hat sich 2013 daher die sogenannte Treaty Alliance gebildet - ein Netzwerk aus mittlerweile über 1000 NGOs, das sich für ein rechtsverbindliches Instrument für multinationale Unternehmen einsetzt. Dem Appell der Treaty Alliance zur Etablierung einer zwischenstaatlichen Arbeitsgruppe für die Ausarbeitung eines internationalen Abkommens zum verbindlichen Schutz von Menschenrechten durch Unternehmen (Treaty Alliance 2013) ist der UN-Menschenrechtsrat (auf Initiative von Ecuador und Südafrika) 2014 gefolgt. Einer der größten Streitpunkte in den laufenden Verhandlungen in der Arbeitsgruppe ist die Frage, ob Unternehmen direkte Verpflichtungen auferlegt werden sollen.

Informationsvermittlung Mit dem Bedeutungszuwachs gesellschaftlicher Verantwortung im unternehmerischen Bewusstsein hat sich um die Jahrtausendwende ein regelrechter Markt gebildet, auf dem NGOs ihre Expertise bereitstellen. Mit Analysen und Beratungsleistungen unterstützen NGOs reformwillige Unternehmen darin, ihre Verantwortung für die Einhaltung der Menschenrechte wahrzunehmen. In diesem Feld sind besonders solche NGOs zu verorten, die einen Think-Tank Charakter aufweisen und eine eher pragmatische Herangehensweise haben. Ein prominentes Beispiel hierfür ist das 2009 gegründete Institute for Business and Human Rights, das Analysen, Berichte und Empfehlungen für Unternehmen aus verschiedenen Sektoren veröffentlicht. Dieses Institut war auch an der Ausarbeitung eines Corporate Human Rights Benchmark beteiligt (Institute for Business and Human Rights 2016). Er bewertet jährlich die größten börsennotierten Unternehmen entlang von 100 Menschenrechtskriterien und soll einen Anreiz für Unternehmen schaffen, sich gegenüber Wettbewerbern als Good Corporate Citizen zu profilieren. Aber auch kritischere Organisationen nutzen Instrumente der Informationsvermittlung wie die Erstellung von Policy Papers oder Studien zu Menschenrechtsverletzungen. Diese sind nicht als Dienstleistung an Unternehmen zu verstehen, sondern richten sich an die Öffentlichkeit und fallen in der Regel weitaus kritischer aus. Beispielhaft ist hier die ausführliche Berichterstattung der NGO Clean Clothes Campaign anlässlich der Sicherheits- und Arbeitsbedingungen in der Textilindustrie zu nennen (Clean Clothes Campaign 2013). Darüber hinaus bieten einige NGOs auch praktische Umsetzungshilfe für Unternehmen an. Ein Beispiel ist in diesem Zusammenhang die NGO Shift. Sie ist auf die individuelle Beratung von Unternehmen spezialisiert, die sich zur Umsetzung von Ruggies Leitprinzipien verpflichtet fühlen (Shift 2021). 
Ko-Regulierung Seit den 2000er Jahren haben einige NGOs die Kooperation mit Unternehmen ausgebaut und verstehen sich als Partnerinnen im Bereich des Menschenrechtsschutzes. Der damalige Gründer und Vorsitzende der Amnesty International UK Business Group, Chandler (2007), beschreibt diese Entwicklung wie folgt: „Those of us who [...] helped to put human rights on the corporate agenda learnt that while protest should never be abandoned, positive engagement, using abuses as a lever for change, not as a beating stick, is the way ahead." Dem Vorgehen von NGOs aus dem Bereich der globalen Umweltpolitik folgend - ,which had long recognised the potential importance of companies and had for many years engaged in dialogue with them“ (Chandler 2009, S. 29), stünden Menschenrechts-NGOs nun auch an der Seite von Unternehmen, um gemeinsam globale Menschenrechtsstandards für die Wirtschaft zu entwickeln. Ein Beispiel hierfür ist die Fair Labor Association (FLA). Sie ist aus einer vom damaligen US-Präsidenten Bill Clinton ins Leben gerufenen Initiative hervorgegangen und wurde 1999 mit dem Ziel gegründet, weltweit Arbeitsrechte zu fördern und zu schützen, sowie Arbeitsbedingungen durch die Einhaltung internationaler Standards zu verbessern. Die Organisation ist eine MSI aus privaten Unternehmen, NGOs und Universitäten. Sie hat einen Workplace Code of Conduct sowie Principles of Monitoring veröffentlicht, die auf Standards der internationalen Arbeitsorganisation basieren. Ihre Zusammensetzung hat sich im Laufe der Zeit stark verändert. So verließen aufgrund der vagen Formulierungen des Codes Gewerkschaftsvertreter die Organisation. Nachdem die Mitgliedsunternehmen zu Beginn vor allem aus der Textil- und Kleidungsindustrie kamen, überprüft die Organisation mittlerweile auch Unternehmen aus der Elektronik- und Landwirtschaftsindustrie, darunter den Industrie-Giganten Apple (Sethi und Rovenpor 2016, S. 13).

Unternehmen, die Mitglied der FLA werden möchten, müssen einen MonitoringPlan einreichen, in dem ihr internes Programm zur Einhaltung der verschiedenen Standards beschrieben wird. Zudem wird eine Strategie gefordert, die es Unternehmen erlaubt, sich an die Prinzipien der FLA zu halten. Über ein Zufallsverfahren in Verbindung mit einer Gefährdungsbeurteilung für Verstöße wird festgelegt, welche Einrichtungen kontrolliert werden. Nach der Einstiegsphase überprüfen unabhängige Kontrolleur*innen fünf Prozent der Geschäftstätigkeiten der teilnehmenden Unternehmen (Fair Labor Association 2014). Während die FLA für die Entwicklung regulativer Instrumente mit technischem Beistand und Unterstützung für Training gelobt wird, kritisieren Beobachter*innen sie vor allem dafür, keine Mechanismen zu nutzen, um Unternehmensanteile auf die Arbeiter zu übertragen. Ein weiterer Kritikpunkt ist die Abhängigkeit von der Finanzierung durch die Unternehmen, welche die Organisation überprüfen (Sethi und Rovenpor 2016, S. 15-16).

Ein Beispiel für eine sektoren-spezifische Kooperation zwischen NGOs, Unternehmen und Regierungsvertretern sind die Voluntary Principles on Security and Human Rights (VPs). Diese MSI wurde im Jahr 2000 geschaffen und richtet sich insbesondere an Unternehmen aus der Petrochemie und der Rohstoff-Industrie, die zu ihrem Schutz auf staatliche und nichtstaatliche Sicherheitskräfte zurückgreifen (Hofferberth 2011, S. 11). Konkret bestehen die VPs aus einer Reihe nicht-bindender Prinzipien, die Unternehmen darin anleiten, Risiken zu evaluieren und Maßnahmen zu treffen, damit bei der Wahrung ihrer Sicherheit durch Sicherheitsfirmen, Militär 
und Polizei die Menschenrechte eingehalten werden. Die Formulierung der Prinzipien war dabei eine Reaktion auf Berichte über die gewaltsame Unterdrückung von Umweltprotesten und willkürlicher Gewalt, die von staatlichen und privaten Sicherheitsdiensten großer Rohstoffunternehmen begangen wurden. Inzwischen wurden die Prinzipien sowohl von Mitgliedern als auch einigen Nicht-Mitgliedern in ihr Management und die Verträge mit Sicherheitsdienstleistern übernommen. Sie beinhalten Klauseln zur regelmäßigen Rücksprache zwischen Unternehmen, Regierung des Gastlandes und der lokalen Bevölkerung sowie zur Überwachung von Menschenrechtsverletzungen. Trotz dieser Bemühungen stehen die VPs in der Kritik, keine glaubwürdige und unabhängige Vor-Ort-Überwachung zu leisten, da Inspektoren zum Teil durch die betroffenen Unternehmen selbst finanziert werden (Greenhouse 2012). Eine Reihe von NGOs, darunter prominente Organisationen wie Amnesty International und Oxfam, haben sich daher aus den VP zurückgezogen (Amnesty International 2013; Oxfam America 2013).

Eine weitere Initiative ist der Global Compact. Er wurde 1999 vom damaligen UN-Generalsekretär Kofi Annan auf dem Weltwirtschaftsforum in Davos ins Leben gerufen und im Folgejahr offiziell gegründet (Williams 2004, S. 755). Ziel des Global Compact war es laut Annan, der Weltwirtschaft ein humaneres Gesicht $\mathrm{zu}$ geben. Um dies zu erreichen, sollten die beteiligten Akteure good practices in den Bereichen Menschenrechte, Arbeiterrechte, Umweltschutz und Anti-Korruption identifizieren und verbreiten. Zu diesem Zweck wurden zehn universelle Prinzipien festgelegt, die die beteiligten Unternehmen in ihrer strategischen und operativen Ausrichtung integrieren sollten (United Nations Clobal Compact 2016). Der Global Compact ist dabei nicht nur als ein Instrument zur Selbstregulierung von Unternehmen gedacht, sondern bietet auch ein Forum, das einen Dialog zwischen einer Vielzahl von Akteuren aus der Zivilgesellschaft und dem Privatsektor ermöglicht (Rasche 2009, S. 6).

\section{NGOs und private Unternehmen in der globalen Sicherheitspolitik}

Informationsvermittlung Als Mitte der 1990er-Jahre sogenannte Private Sicherheits- und Militärfirmen (PSMFs) in der globalen Sicherheitspolitik an Bedeutung gewannen, konzentrierten sich die in diesem Bereich tätigen NGOs zunächst auf Strategien der Politikberatung gegenüber PSMFs und Entscheidungsträger*innen. Nachdem 1998 öffentlich wurde, dass die britische Firma Sandline International unter Umgehung eines UN-Waffenembargos Kriegsgerät nach Sierra Leone geliefert hatte, entbrannte in Großbritannien eine Diskussion zur stärkeren Regulierung und Kontrolle von PSMFs (Kinsey 2006). Dieser Prozess mündete 2002 in der Veröffentlichung eines Grünbuchs, in dem die britische Regierung unterschiedliche Optionen zur Regulierung der Branche darlegte. Angefacht durch das aufkommende Interesse in die Sicherheitsbranche, schalteten sich NGOs wie International Alert und Saferworld in die Privatisierungs-Debatte ein. Der Fokus ihrer Arbeit lag dabei weniger auf der Skandalisierung unternehmerischen Fehlverhaltens. Vielmehr haben sich die beiden NGOs auf Strategien der Politikberatung beschränkt, die auf eine gezielte Einflussnahme auf den nationalen Regulierungsprozess gerichtet wa- 
ren. Dazu zählte die Veröffentlichung von ausführlichen Policy-Berichten, die in das Konsultationsverfahren im Grünbuch-Prozess einflossen (Makki et al. 2001; Lilly 2002).

Auffällig sind der neutrale Ton, den beide NGOs anschlugen und ihre ausgewogene, sachliche Darstellungsweise der Sicherheitsbranche. Zwar wiesen sie auf die potenziellen Gefahren dieser Firmen für Frieden, Stabilität und den Schutz von Menschenrechten hin, hielten sich aber in ihrer Kritik gegenüber PSMFs merklich zurück (Beyani und Lilly 2001; Lilly 2000). Angesichts des Kräftemangels afrikanischer Streitkräfte und der fehlenden Interventionsbereitschaft westlicher Staaten, stellten PSMFs aus ihrer Sicht eine legitime Alternative dar, um Frieden und Sicherheit wiederherzustellen und den Zerfall von Staaten zu verhindern (Lilly 2000, S. 12).

Das Internationale Komitee vom Roten Kreuz verfolgte einen ähnlichen Ansatz. Seit 2004 hat sich die NGO dem Thema der Privatisierung von Sicherheit angenommen, dabei aber explizit Naming and Shaming-Kampagnen als Strategie ausgeschlossen. Stattdessen strebte die Organisation an, durch einen konstruktiven Dialog mit den größten Sicherheitsfirmen existierende Unternehmenspraktiken zu verbessern und nachteilige humanitäre Auswirkungen zu reduzieren (ICRC 2004; Voillat 2012, S. 1093). Diese pragmatische Herangehensweise trug dem Mandat des Roten Kreuzes Rechnung. Um in kritischen Einsatzgebieten operieren zu können, ist die Organisation auf die Akzeptanz aller Parteien angewiesen, auch der privaten Sicherheitsdienstleister, was sich in ihrem Bemühen um eine unpolitische und unparteiische Position niederschlägt.

Agenda-Setting Der massive Einsatz von PSMFs im Irak und in Afghanistan in den 2000er Jahren hat die NGO-Community zu einer Neubewertung ihrer Strategie gegenüber der Branche bewogen. Nicht nur zahlenmäßig sind PSMFs von einem Randphänomen in krisengeschüttelten afrikanischen Staaten zu einem wichtigen Faktor aufgestiegen (Stürchler 2008, S. 10). Gleichzeitig stießen medienwirksame Vorfälle, in die Mitarbeiter*innen von Sicherheitsdienstleistern verwickelt waren, eine erneute Diskussion des Themas an. Seither machen NGOs wie Amnesty International, Human Rights First, War on Want und Campaign Against the Arms Trade vermehrt in unternehmenskritischen Kampagnen auf die Schattenseiten der Privatisierung der globalen Sicherheitspolitik aufmerksam. Sie prangerten Menschenrechtsverletzungen durch PSMFs an, betonten ihre Rolle als Profiteure von Krieg, Instabilität und Unsicherheit und kritisierten die Erosion des staatlichen Gewaltmonopols durch die Auslagerung von Kernkompetenzen an diese Unternehmen (Prem 2020).

Vor diesem Hintergrund griffen NGOs auf Naming and Shaming-Kampagnen gegen prominente PSMFs zurück. Im Schlaglicht solcher Kampagnen standen insbesondere zwei Unternehmen: Blackwater und Titan. Seit dem Skandal um die Misshandlung von Häftlingen im Militärgefängnis Abu Ghraib durch Mitarbeiter*innen der Firma Titan und dem Nissour-Square-Vorfall, bei dem Blackwater-Angestellte willkürlich auf Zivilist*innen schossen und 17 von ihnen töteten, haftet ihnen der Ruf als schwarze Schafe der Branche an. Darüber hinaus wurden große Markenunternehmen wie G4S ins Visier genommen (War on Want 2008, 2012, 2013, 
2014). Seit der Übernahme von ArmorGroup 2008 ist G4S die weltgrößte private Sicherheitsfirma, was sie zu einem sehr sichtbaren und damit potenziell verletzbaren Gegner macht (Yaziji und Doh 2009, S. 99; Flohr et al. 2010).

Die Skandalisierung der Praktiken einzelner PSMFs kann aber auch als Warnschuss für den gesamten Sektor verstanden werden (Coni-Zimmer 2012, S. 322). NGOs nutzten diese Skandale, um auf Problemlagen aufmerksam zu machen, die beispielhaft für die gesamte Sicherheitsbranche sind. Sie bemühten sich darauf hinzuweisen, dass der willkürliche Einsatz von Gewalt durch Angestellte dieser Firmen keine Einzelfälle darstelle, sondern lediglich die Spitze des Eisberges sei (Human Rights First 2008, S. 2). Indem sie Menschenrechtsverletzungen durch Sicherheitsunternehmen als strukturelles Problem deklarierten, verliehen NGOs ihrer Forderung nach einer staatlichen Regulierung der Branche Nachdruck. Dementsprechend richteten NGOs ihre Forderungen häufig auch an die Staaten, in denen diese Firmen registriert waren und/oder die als ihre Aufraggeber agierten - allen voran die USA und Großbritannien. Ihnen wurde die Hauptverantwortung für die Regulierung der Sicherheitsbranche zugeschrieben (Chatterjee 2008, S. 25).

Dabei ist auffällig, dass der Forderungskatalog, den NGOs an die Regierungen und Parlamente der beiden Staaten herantrugen, vergleichsweise moderat ausfiel. Statt wie in der Shell-Kampagne zu Boykotten aufzurufen oder gar ein Ende der Privatisierung von Sicherheitsleistungen zu fordern, zielten die meisten NGOs mit ihren Vorschlägen auf eine Verbesserung des regulativen Umfelds, in dem sich PSMF bewegten. Ihre Rolle als zentrale Akteure der globalen Sicherheitsarchitektur wurde dagegen nur vereinzelt infrage gestellt. Auch zahlenmäßig sind kritische Kampagnen von NGOs gegen PSMF eher eine Randerscheinung geblieben (Pingeot 2014, S. 15). Öffentliche Protestaktivitäten wie anlässlich der Jahreshauptversammlung der British Association of Private Security Companies, als Aktivist*innen in Söldneruniformen verkleidet gegen einen freiwilligen Verhaltenskodex demonstrierten, bildeten eher die Ausnahme. Große NGOs wie Amnesty International und Human Rights First, denen in der Literatur häufig eine Signalwirkung im Agenda-SettingProzess zugesprochen wird (Carpenter 2007b, S. 103), haben die Veröffentlichung von kritischen Berichten über Sicherheitsfirmen zugunsten ihrer Mitwirkung in gemeinsamen Regelsetzungsprozessen in den letzten Jahren ganz eingestellt.

Ko-Regulierung Ein frühes Beispiel für die institutionalisierte Zusammenarbeit von NGOs und privaten Sicherheitsunternehmen ist die Schweizer Initiative. Sie geht auf Bestreben der Schweizer Regierung zurück, die 2006 zusammen mit dem Roten Kreuz einen Aushandlungsprozess zur Klärung und Konkretisierung bestehender völkerrechtlicher Verpflichtungen angestoßen hat. Diese gelten, wenn private Sicherheitsfirmen in bewaffneten Konflikten operieren. Aus diesem Prozess ist 2008 das sogenannte Montreux-Dokument hervorgegangen, ein rechtlich nicht-bindender Katalog, der bereits bestehende und für den Einsatz von Sicherheitsfirmen relevante Verpflichtungen aus geltendem Völkerrecht zusammenträgt. In einem zweiten Teil gibt er außerdem Empfehlungen in Form von 70 Good Practices ab, wie diese Staaten ihre völkerrechtlichen Verpflichtungen auf nationaler Ebene umsetzen können. Bei der Ausarbeitung des Dokuments nahmen neben Regierungsmitarbeiter*innen auch Industrievertreter*innen und zivilgesellschaftliche Gruppen teil. Der Einbe- 
zug nichtstaatlicher Akteure hatte dabei in erster Linie konsultativen Charakter; sie waren zwar im Aushandlungsprozess beratend tätig, hatten aber keinen formellen Einfluss auf die Entscheidungsfindung.

Obwohl zivilgesellschaftliche Organisationen diesen Prozess ausdrücklich begrüßten, bestand im Lager der NGOs Einigkeit, dass weitere Schritte notwendig waren, um verantwortliches Unternehmenshandeln zu fördern (Buzatu 2015, S. 27). Dabei beklagten NGOs den langsamen Fortschritt zu einer gesetzlichen Regulierung. Aus ihrer Sicht hatten staatliche Akteure schlicht kein Interesse an einem verbindlichen Abkommen, da sie in hohem Maße von ihren Leistungen in Konfliktgebieten abhängig waren (Schwartz 2011, S. 11) oder vom Export der Sicherheitsdienstleistungen profitierten (Clive und Dave 2005). Aus dieser Einsicht heraus ist mit dem Prozess zur Etablierung des Internationalen Verhaltenskodexes für private Sicherheitsunternehmen (ICoC) eine weitere MSI entstanden. Dieser Kodex ist seit 2012 gültig und richtet sich direkt an PSMF, die sich mit seiner Unterzeichnung zur Achtung von Menschenrechten und des humanitären Völkerrechts verpflichten. Er formuliert darüber hinaus Managementrichtlinien, die zur Einhaltung seiner Grundsätze beitragen sollen, sowie Standards für die Rekrutierung und Weiterbildung von Sicherheitspersonal. Anders als in der Schweizer Initiative bewegten sich NGOs bei diesem Prozess auf Augenhöhe mit Regierungsvertretern und privaten Sicherheitsfirmen, da sie unmittelbar in die Entscheidungsprozesse einbezogen waren.

Diese Struktur spiegelt sich auch in der Folgeinitiative wider. Nach dem Abschluss der Verhandlungen wurde ein aus den drei Sektoren - Regierungen, Privatsektor und Zivilgesellschaft - zusammengesetztes Steuerungskomitee damit beauftragt, ein Kontrollorgan zu erarbeiten: die Vereinigung des internationalen Verhaltenskodex für private Sicherheitsdienstleister (ICoCA). Es soll die Umsetzung der im Kodex festgehaltenen Regeln gewährleisten. Durch ihre Beteiligung am Lenkungsausschuss der Vereinigung nehmen NGOs eine neue Überwachungs- und Kontrollfunktion gegenüber privaten Sicherheitsfirmen wahr. Sie sind in der Lage, Firmen zum Zertifizierungsprozess zuzulassen, Normverstöße von Unternehmen festzustellen und korrigierende Maßnahmen anzustoßen. Anders als etwa in der Umweltpolitik sind sie jedoch nicht direkt an der Umsetzung des Kodex involviert. Um die Unabhängigkeit der zivilgesellschaftlichen Säule in der Vereinigung zu bewahren, waren die an der ICoCA beteiligten NGOs darauf bedacht, die Teilnahme von zivilgesellschaftlichen Organisationen auszuschließen, die als Beraterinnen oder Implementierungshelferinnen für PSMFs tätig sind (Prem 2020, S. 161). Sogenannte consultanttype NGOs haben sich außerhalb der Governance-Strukturen der ICoCA darauf spezialisiert, Trainings- und Beratungsleistungen zur Umsetzung des Verhaltenskodex anzubieten.

Gleichzeitig gibt es aber auch NGOs wie War on Want, die den Trend zur Zusammenarbeit mit PSMF verweigern. Diese fallen durch radikale Forderungen wie eine Abkehr von bestehenden Privatisierungspraktiken auf und unterstützen die vom UN-Menschenrechtsrat ins Leben gerufene Arbeitsgruppe, deren Ziel die Ausarbeitung eines rechtliche bindendes Regelwerk für PSMF ist. Allerdings ist es nach der Etablierung der Schweizer Initiative und dem ICoC für solche kritischen Stimmen schwieriger geworden, Gehör für ihre Anliegen zu finden (Prem 2021). Nach Ansicht einiger Autoren nimmt stattdessen der systemische Druck auf zivilgesellschaftliche 
Organisationen zu, mit der Privatwirtschaft zu kooperieren (Coni-Zimmer und Flohr 2015, S. 582).

\section{Fazit}

Mit Blick auf unsere eingangs formulierte Frage lässt sich über die drei Politikfelder hinweg eine konvergente Entwicklung im Verhältnis von NGOs und Unternehmen beobachten: Seit den 1980er/1990er-Jahren haben NGOs ihre bis dahin dominierende Strategie als Agenda-Setter und Informationsvermittler gegenüber Unternehmen erweitert und beteiligen sich nun vielfach mit Akteuren aus der Privatwirtschaft an der Regelsetzung, Überwachung und Implementierung in MSIs. Die Umweltpolitik wird zwar häufig als Paradebeispiel für diese Entwicklung angeführt. Unsere Studie macht jedoch deutlich, dass auch in der Menschenrechts- und Sicherheitspolitik öffentliche, private und zivilgesellschaftliche Akteure in gemeinsamen GovernanceStrukturen an Lösungen für globale Probleme arbeiten.

Gleichzeitig lassen sich auch Unterschiede beim Vergleich der Politikfelder erkennen. Die Funktionserweiterung von NGOs gegenüber Unternehmen verläuft je nach Politikfeld zeitlich versetzt und ist in den einzelnen Dimensionen unterschiedlich stark ausgeprägt. Anders als in der Umwelt- und Menschenrechtspolitik traten NGOs im Bereich der privaten Sicherheitspolitik zum Beispiel erst als Informationsvermittler auf, indem sie Staaten aber auch private Sicherheitsunternehmen bei ihrer Tätigkeit beratend unterstützten. Erst nachdem einige Skandale und Fehlverhalten privater Sicherheitsdienstleister bekannt wurden, formierten sich in Teilen des NGO-Lagers Protestbewegungen gegen die Privatisierung von Sicherheit. Auffällig ist zudem, dass im Vergleich zur globalen Umwelt- und Menschrechtspolitik die Kritik von NGOs gegenüber dem Sicherheitssektor von Beginn an moderater ausfällt. Boykottaufrufe und öffentliche Protestaktivitäten wie in der Shell- und Anti-Sweatshop-Kampagne blieben weitgehend aus. Wie wir im folgenden Abschnitt weiter ausführen, könnte dieser Umstand auf die Diffusion eines globalen Skripts zurückgeführt werden, das konfrontative Strategien zunehmend delegitimiert. Aber auch politikfeldspezifische Faktoren spielen eine Rolle. Anders als verbraucherorientierte Unternehmen sind private Sicherheitsfirmen weniger anfällig für solche Proteste, da ihre Kunden keine Individuen, sondern Staaten und andere Unternehmen sind. Deren ,Kaufentscheidungen“ basieren auf Sicherheitsinteressen und Kosten-Nutzen-Berechnungen und sind weniger durch normative Bedenken beeinflusst (Cockayne 2008, S. 428; Krahmann 2016). Zudem hat die Nutzung privater Sicherheitsdienstleistungen durch eine Reihe humanitärer Organisationen selbst eine zähmende Wirkung auf die betreffenden NGOs (Joachim und Schneiker 2015). Die größten NGOs gaben ihre kritische Haltung zugunsten ihrer Mitwirkung in gemeinsamen Regelsetzungsprozessen sogar komplett auf. Daher lässt sich auch in der Sicherheitspolitik ein zunehmender Rollenwandel von NGOs konstatieren.

Die Gründe für diese Entwicklung sind vielfältig und bedürfen weiterer Forschung. Eine zentrale Erklärungsvariante für den Rollenwandel von NGOs in ihrem Verhältnis gegenüber der Privatwirtschaft ist die zunehmende Professionalisierung von NGOs (Lang 2012). Andere Autor*innen betonen die Ressourcenbasis oder 
Opportunitätsstrukturen der jeweiligen NGOs (Yaziji und Doh 2009). Unsere Beobachtung eines politikfeldübergreifenden Trends legt allerdings die Vermutung nahe, dass die Funktionserweiterung von NGOs weniger auf der Ebene der individuellen Organisationen zu verorten ist, als auf struktureller Ebene. Neo-institutionelle Theorien wie der soziologische Institutionalismus gehen beispielsweise davon aus, dass organisationaler Wandel durch Veränderungen im normativen Umfeld verursacht wird (Meyer und Rowan 1977). Vorherrschende Normen und kognitive Skripte können zur Herausbildung gleichförmiger Organisationsformen und -praktiken (Isomorphismus) führen (DiMaggio und Powell 1983), wie wir am Beispiel der politikfeldübergreifenden Funktionserweiterung von NGOs beobachten konnten. In diesem Sinne kann das sich wandelnde Verhältnis zwischen NGOs und dem Privatsektor als Diffusion eines globalen Skripts begriffen werden, das eine aktive Einbindung nichtstaatlicher Akteure in die Politikgestaltung propagiert, um grenzüberschreitende Probleme zu lösen (Coni-Zimmer und Flohr 2015, S. 582). Wir stellen daher die Hypothese auf, dass es in allen drei untersuchten Politikfeldern eine zunehmende Erwartungshaltung gegenüber NGOs gibt, eine ko-regulierende und auf partnerschaftliche Zusammenarbeit mit dem Privatsektor ausgelegte Rolle einzunehmen. Diese Norm fand erstmals auf der Rio-Konferenz Einzug in die internationale Umweltpolitik und wurde in Organisationen wie der ISEAL-Alliance, dem FSC und dem MSC institutionalisiert. Diese Initiativen zeigten, dass sektoren-übergreifende Partnerschaften nicht nur möglich waren, sondern zunehmend als effektiver und legitimer Weg zur Förderung von Verhaltensänderungen in Unternehmen angesehen wurden (Pattberg 2005, S. 182). Partnerschaften mit Unternehmen wurden zu einem „Modell“, das auch NGOs in anderen Politikfeldern nachahmten, wie am Beispiel der Menschenrechts- und Sicherheitspolitik erkennbar wird.

Empirisch lässt sich der von uns diagnostizierte Trend in die Governance-Forschung einbetten. Angesichts der zunehmenden Öffnung von internationalen Organisationen gegenüber nicht-staatlichen Akteuren und der Proliferation neuer Governance-Formen (MSIs, globale Politiknetzwerke und Public-Private-Partnerships) sprechen einige Autor*innen von einer neuen Norm, die einen pluralistischen und partnerschaftlichen Ansatz des globalen Regierens vorsieht (Dingwerth und Pattberg 2009, S. 731; Liese 2009; Reimann 2006; Tallberg 2010). In der Literatur wird dieser Trend auf die im UN-System verbreitete Norm der partnerschaftlichen Zusammenarbeit zwischen den drei Sektoren - Staat, Zivilgesellschaft und Privatsektor zurückgeführt (Böhling 2011). Damit einher ging nicht nur eine Stärkung nichtstaatlicher Akteure im globalen Regieren (Dany 2014). Für viele NGOs wurden die Weltkonferenzen ebenfalls zum Anlass, ihre klassische Watchdog Rolle gegenüber privaten Unternehmen zu erweitern (Utting 2000, S. 6).

Die Funktionserweiterung von NGOs wird in der Literatur kontrovers diskutiert. Einerseits eröffnet sie NGOs großen Einfluss- und Handlungsspielraum, um das Handeln von Unternehmen und Staaten zu verändern (Betsill und Corell 2008). Andererseits halten viele Autor*innen diesen Einfluss aufgrund beschränkter Zugangsmöglichkeiten für begrenzt (Dany 2014). Sie weisen ebenfalls darauf hin, dass NGOs in Interessenskonflikte geraten und ihre Autonomie, Glaubwürdigkeit und Kritikfähigkeit gegenüber dem Privatsektor aufs Spiel setzen können (Baur und Schmitz 2012; Joachim und Schneiker 2015). Angesichts dieser Prognosen bleibt 
zu prüfen, inwieweit sich NGOs dem Druck zur partnerschaftlichen Zusammenarbeit widersetzen (können). Unsere Analyse gibt erste Hinweise darauf, dass der von uns konstatierte Trend zur Ko-Regulierung weder absolut noch unumkehrbar ist. In allen Politikfeldern gibt es weiterhin kritische NGOs, die eine Mitarbeit in MSI ablehnen und sich für verbindliche Regeln einsetzen. Der Rückzug von Partnerschaften grundsätzlich aufgeschlossenen Organisationen wie Greenpeace, Amnesty International und Oxfam aus etablierten MSI spricht gar für eine gewisse „MultiStakeholder Müdigkeit“".

Funding Open access funding provided by Utrecht University.

Open Access Dieser Artikel wird unter der Creative Commons Namensnennung 4.0 International Lizenz veröffentlicht, welche die Nutzung, Vervielfältigung, Bearbeitung, Verbreitung und Wiedergabe in jeglichem Medium und Format erlaubt, sofern Sie den/die ursprünglichen Autor(en) und die Quelle ordnungsgemäß nennen, einen Link zur Creative Commons Lizenz beifügen und angeben, ob Änderungen vorgenommen wurden.

Die in diesem Artikel enthaltenen Bilder und sonstiges Drittmaterial unterliegen ebenfalls der genannten Creative Commons Lizenz, sofern sich aus der Abbildungslegende nichts anderes ergibt. Sofern das betreffende Material nicht unter der genannten Creative Commons Lizenz steht und die betreffende Handlung nicht nach gesetzlichen Vorschriften erlaubt ist, ist für die oben aufgeführten Weiterverwendungen des Materials die Einwilligung des jeweiligen Rechteinhabers einzuholen.

Weitere Details zur Lizenz entnehmen Sie bitte der Lizenzinformation auf http://creativecommons.org/ licenses/by/4.0/deed.de.

\section{Literatur}

Abbott, Kenneth W., und Duncan Snidal. 2009. The governance triangle: regulatory standards institutions and the shadow of the state. In The politics of global regulation, Hrsg. Ngaire Woods, Walter Mattli, 44-88. Princeton: Princeton University Press.

Albin-Lackey, Chris. 2013. Without rules. A failed approach to corporate accountability. https://www.hrw. org/sites/default/files/related_material/business.pdf. Zugegriffen: 15. März 2021.

Amnesty International. 2013. Amnesty international withdrawal from the voluntary principles on security and human rights. https://www.amnesty.org/download/Documents/16000/ior400032013en.pdf. Zugegriffen: 24. März 2021.

Andonova, Liliana B. 2010. Public-private partnerships for the earth: politics and patterns of hybrid authority in the multilateral system. Global Environmental Politics 10(2):25-53.

Argenti, Paul A. 2004. Collaborating with activists: how Starbucks works with NGOs. California Management Review 47(1):91-116.

Avant, Deborah D., Martha Finnemore, und Susan K. Sell. 2010. Who governs the globe? In Who governs the globe? Cambridge studies in international relations, Hrsg. Deborah D. Avant, Martha Finnemore, und Susan K. Sell, 1-31. Cambridge: Cambridge University Press.

Bäckstrand, Karin, Jamil Khan, Annica Kronsell, und Eva Lövbrand. 2010. Environmental politics and deliberative democracy: examining the promise of new modes of governance. Cheltenham: Edward Elgar.

Bartley, Tim. 2014. Transnational governance and the re-centered state: sustainability or legality? Regulation \& Governance 8(1):93-109.

Baumann-Pauly, Dorothée, Justine Nolan, Auret van Heerden, und Michael Samway. 2016. Industry-specific multi-stakeholder initiatives that govern corporate human rights standards: legitimacy assessments of the fair labor association and the global network initiative. Journal of Business Ethics 143:771-787

Baur, Dorothea, und Hans Peter Schmitz. 2012. Corporations and NGOs: when accountability leads to cooptation. Journal of Business Ethics 106(1):9-21.

Bell, Stephen, und Andrew Hindmoor. 2012. Governance without government? The case of the forest stewardship council. Public Administration 90(1):144-159. 
Bendell, Jem. 2000. Working with Stakeholder Pressure for Sustainable Development. In Terms for Endearment: Business, NGOs and Sustainable Development, Hrsg. Jem Bendell, David F. Murphy, 14-30. Sheffield: Greenleaf.

Bennie, Lynn G. 1998. Brent Spar, atlantic oil and Greenpeace. Parliamentary Affairs 51(3):397-410.

Betsill, Michele, und Elisabeth Corell. 2008. NGO diplomacy: the influence of nongovernmental organizations in international environmental negotiations. Cambridge: MIT Press.

Beyani, Chaloka, und Damian Lilly. 2001. Regulating private military companies. Options for the UK government. London: International Alert.

Böhling, Kathrin. 2011. Multistakeholder involvement in UN conferences. In The handbook of transnational governance. Institutions and innovations, Hrsg. Thomas Hale, David Held, 195-202. Cambridge: Polity Press.

Börzel, Tanja A., und Thomas Risse. 2005. Public-private partnerships: effective and legitimate tools of international governance. In Complex sovereignty: reconstituting political authority in the twentyfirst century, Hrsg. Edgar Grande, Louis W. Pauly, 195-216. Toronto: University of Toronto Press.

Börzel, Tanja A., und Thomas Risse. 2007. Public-private partnerships: effective and legitimate tools of transnational governance? In Complex sovereignty: reconstituting political authority in the twentyfirst century, Hrsg. Louis W. Pauly, Edgar Grande, 195-216. Toronto: University of Toronto Press.

Boström, Magnus, und Kristina Tamm Hallström. 2010. NGO power in global social and environmental standard-setting. Global Environmental Politics 10(4):36-59.

Buzatu, Anne-Marie. 2015. Towards an international code of conduct for private security providers: a view from inside a multistakeholder process. Geneva: Centre for the Democratic Control of Armed Forces.

Carpenter, R. Charli. 2007a. Setting the advocacy agenda: theorizing issue emergence and nonemergence in transnational advocacy networks. International Studies Quarterly 51(1):99-120.

Carpenter, R. Charli. 2007b. Studying issue (non)-adoption in transnational advocacy networks. International Organization 61(3):643-667.

Carty, Victoria. 2001. The Internet and grassroots politics: nike, the athletic apparel industry and the antisweatshop campaign. Tamara Journal for Critical Organization Inquiry 1(2):34-48.

Chandler, Geoffrey. 2007. Open letter to the signatories of the 10 October letter to the Special Representative of the UN Secretary General for business and human rights. 15. Oktober. https://www. business-humanrights.org/en/latest-news/submissions-to-un-special-representative-john-ruggieand-responses. Zugegriffen: 23. Juni 2021

Chandler, Geoffrey. 2009. The Amnesty International UK business group: putting human rights on the corporate agenda. The Journal of Corporate Citizenship 33:29-34.

Chatterjee, Pratap. 2008. Outsourcing intelligence in Iraq. A Corpwatch report on L-3/titan. Oakland: Corpwatch and Amnesty International.

Clapp, Jennifer. 1998. The privatization of global environmental governance: ISO 14000 and the developing world. Global Governance 4(3):295-316.

Clapp, Jennifer. 2005. Global environmental governance for corporate responsibility and accountability. Global Environmental Politics 5(3):23-34.

Clean Clothes Campaign. 2013. Rana plaza: a man-made disaster that shook the world. https://web.archive. org/web/20150424134515/http://www.cleanclothes.org/ua/2013/rana-plaza. Zugegriffen: 25. März 2021.

Clive, Walker, und Whyte Dave. 2005. Contracting out war? Private military companies, law and regulation in the United Kingdom. International \& Comparative Law Quarterly 54(03):651-689.

Cockayne, James. 2008. Regulating private military and security companies: the content, negotiation, weaknesses and promise of the Montreux document. Journal of Conflict and Security Law 13(3):401-428.

Conca, Ken. 1995. Greening the United Nations: environmental organizations and the UN system. Third World Quarterly 16(3):441-457.

Coni-Zimmer, Melanie. 2012. Zivilgesellschaftliche Kritik und CSR als unternehmerische Legitimitätspolitik. In Der Aufstieg der Legitimitätspolitik. Rechtfertigung und Kritik politisch-ökonomischer Ordnungen, Hrsg. Anna Geis, Frank Nullmeier, und Christopher Daase, 319-336. Baden-Baden: Nomos.

Coni-Zimmer, Melanie, und Annegret Flohr. 2015. Zwischen Konfrontation und Kooperation - Das Verhältnis zwischen NGOs und Privatwirtschaft. Zeitschrift für Außen- und Sicherheitspolitik 8(2):567-588.

Curbach, Janina. 2008. Zwischen Boykott und CSR - Eine Beziehungsanalyse zu Unternehmen und NGOs. Journal for Business, Economics and Ethics 9(3):368-395. 
Cutler, A. Claire, Virginia Haufler, und Tony Porter. 1999. Private authority and international affairs. In Private authority and international affairs, Hrsg. A. Claire Cutler, Virginia Haufler, und Tony Porter, 3-28. Albany: State University of New York Press.

Dany, Charlotte. 2014. Janus-faced NGO participation in global governance: structural constrains for NGO influence. Global Governance 20(3):419-436.

DeWinter-Schmitt, Rebecca. 2007. Business as usual? The mobilization of the anti-sweatshop movement and the social construction of corporate identity. Washington D.C.: American University.

DiMaggio, Paul J., und Walter W. Powell. 1983. The iron cage revisited: institutional isomorphism and collective rationality in organizational fields. American Sociological Review 48(2):147-160.

Dingwerth, Klaus. 2008. North-south parity in global governance: the affirmative procedures of the Forest Stewardship Council. Global Governance: A Review of Multilateralism and International Organizations 14(1):53-71.

Dingwerth, Klaus, und Philipp Pattberg. 2009. World politics and organizational fields: the case of transnational sustainability governance. European Journal of International Relations 15(4):707-743.

Eberlein, Burkard Kenneth W.Abbott, Julia Black, und Stephan Wood. 2014. Transnational business governance interactions: conceptualization and framework for analysis. Regulation and Governance 2014(8):1-21.

Entine, Jon. 2002. Shell, Greenpeace and Brent Spar: the politics of dialogue. In Case histories in business ethics, Hrsg. Chris Megone, Simon J. Robinson, 59-95. London: Routledge.

Fair Labor Association. 2014. Charter document. Washington: Fair Labor Association.

Falkner, Robert. 2003. Private environmental governance and international relations: exploring the links. Global Environmental Politics 3(2):72-87.

Flohr, Annegret, Lothar Rieth, Sandra Schwindenhammer, und Klaus Dieter Wolf. 2010. The role of business in global governance: corporations as norm-entrepreneurs. Global issues series. Basingstoke: Palgrave Macmillan.

Greenhouse, Steven. 2012. Critics question record of monitor selected by Apple. New York Times, 13 Februar.

Greenpeace. 2018. Greenpeace international to not renew FSC membership. https://www.greenpeace.org/ international/press-release/15589/greenpeace-international-to-not-renew-fsc-membership/. Zugegriffen: 15. März 2021.

Greenpeace. 2021. Destruction certified. https://www.greenpeace.org/static/planet4-international-stateless/ 2021/03/f66b926f-destruction_certified_09_03_21.pdf. Zugegriffen: 15. März 2021.

Gulbrandsen, Lars H. 2004. Overlapping public and private governance: can forest certification fill the gaps in the global forest regime? Global Environmental Politics 4(2):75-99.

Gulbrandsen, Lars H. 2010. Transnational environmental governance: the emergence and effects of the certification of forests and fisheries. Cheltenham: Edward Elgar.

Hall, Rodney Bruce, und Thomas J. Biersteker. 2002. The emergence of private authority in global governance. Cambridge: Cambridge University Press.

Hamm, Brigitte. 2016. Die UN-Leitprinzipien für Wirtschaft und Menschenrechte: Auswirkungen auf das Menschenrechtsregime. Kritische Justiz 49(4):479-495.

Harrison, Ann, und Jason Scorse. 2010. Multinationals and anti-sweatshop activism. American Economic Review 100(1):247-273.

Hayhurst, Lyndsay M.C., und Courtney Szto. 2016. Corporatizating activism through sport-focused social justice? Investigating Nike's corporate responsibility in sport for development and peace. Journal of Sport and Social Issues 40(6):522-544.

Hickmann, Thomas. 2017. Voluntary global business initiatives and the international climate negotiations: a case study of the Greenhouse Gas Protocol. Journal of Cleaner Production 169:94-104.

Hickmann, Thomas, und Berenike Prem. 2018. Von Gegnern zu Partnern? Zum Verhältnis von NGOs und Unternehmen in der internationalen Klimapolitik. Aus Politik und Zeitgeschichte 68(21-23):48-54.

Hofferberth, Matthias. 2011. The binding dynamics of non-binding governance arrangements. The voluntary principles on security and human rights and the cases of BP and Chevron. Business and Politics 13(4): $1-32$.

van Huijstee, Mariëtte, und Pieter Glasbergen. 2010. NGOs moving business: an analysis of contrasting strategies. Business \& Society 49(4):591-618.

Human Rights First. 2008. Private security contractors at war. Ending the culture of impunity. http://www. humanrightsfirst.org/wp-content/uploads/pdf/08115-usls-psc-final.pdf. Zugegriffen: 24. März 2021.

ICRC. 2004. The ICRC to expand contacts with private military and security companies. http://www.icrc. org/eng/resources/documents/misc/63he58.htm. Zugegriffen: 24. März 2021. 
Institute for Business and Human Rights. 2016. Benchmarking. https:/www.ihrb.org/focus-areas/ benchmarking. Zugegriffen: 26. Nov. 2016.

Joachim, Jutta, und Andrea Schneiker. 2015. NGOs and the price of governance: the trade-offs between regulating and criticizing private military and security companies. Critical Military Studies 1(3):185-201.

Jordan, Grant. 1998. Indirect causes and effects in policy change: the Brent Spar case. Public Administration 76(4):713-740.

Jordan, Grant. 2001. Shell, Greenpeace and the Brent Spar. Basingstoke: Palgrave.

Kalfagianni, Agni, und Philipp Pattberg. 2013. Global fisheries governance beyond the state: unraveling the effectiveness of the Marine Stewardship Council. Journal of Environmental Studies and Sciences 3(2):184-193.

Keck, Margaret E., und Kathryn Sikkink. 1998. Activists beyond borders. Advocacy networks in international politics. Ithaca: Cornell University Press.

Kinsey, Christopher. 2006. Corporate soldiers and international security: the rise of private military companies. London: Routledge.

Klotz, Audie. 1995. Norms in international relations: the struggle against apartheid. Cornell studies in political economy. Ithaca: Cornell University Press.

Krahmann, Elke. 2016. Choice, voice, and exit: consumer power and the self-regulation of the private security industry. European Journal of International Security 1(01):27-48.

Lang, Sabine. 2012. NGOs, civil society, and the public sphere. Cambridge: Cambridge University Press.

Liese, Andrea. 2009. Die unterschiedlich starke Öffnung internationaler Organisationen gegenüber nichtstaatlichen Akteuren. Erklärungen der Institutionen- und Organisationstheorie. In Die Organisierte Welt: Internationale Beziehungen und Organisationsforschung, Hrsg. Klaus Dingwerth, Dieter Kerwer, und Andreas Nölke, 189-210. Baden-Baden: Nomos.

Lilly, Damian. 2000. The privatization of security and peacebuilding: a framework for action. London: International Alert, Policy and Advocacy Department.

Lilly, Damian. 2002. Regulating private military companies: the need for a multidimensional approach. London: International Alert.

Loconto, Allison, und Eve Fouilleux. 2014. Politics of private regulation: ISEAL and the shaping of transnational sustainability governance. Regulation \& Governance 8(2):166-185.

Makki, Sami, Sarah Meek, Abdel-Fatau Musah, Michael Crowley, und Damian Lilly. 2001. Private military companies and the proliferation of small arms: regulating the actors. London: British American Security Information Councel, International Alert and Saferworld.

Meyer, John W., und Brian Rowan. 1977. Institutionalized organizations: formal structure as myth and ceremony. American Journal of Sociology 83(2):340-363.

Moser, Christine, und Sina Leipold. 2021. Toward "hardened" accountability? Analyzing the European Union's hybrid transnational governance in timber and biofuel supply chains. Regulation \& Governance 15(1):115-132.

Mühle, Ursula. 2010. The politics of corporate social responsibility: the rise of a global business norm. Frankfurt am Main: Campus.

Murphy, David F., und David Mathew. 2001. Nike and global labour practices. A case study prepared for the new academy of business innovation network for socially responsible business. http://www. adapttech.it/old/files/document/5282NGLP_2009.pdf. Zugegriffen: 28. Febr. 2017.

Nash, Nathaniel C. (1995). Oil Companies Face Boycott Over Sinking of Rig. New York Times, 17 Juni.

Newell, Peter. 2000. Environmental NGOs and globalization: the governance of TNCs. In Global social movements, Hrsg. Robin Cohen, Shirin M. Rai, 117-132. London: Athlone Press.

Oxfam America. 2013. Oxfam leaves voluntary principles for security and human rights multi-stakeholder initiative. http://web.archive.org/web/20131006092902/http://www.oxfamamerica.org/press/ pressreleases/oxfam-leaves-voluntary-principles-for-security-and-human-rights-multi-stakeholderinitiative-1/. Zugegriffen: 2. Febr. 2016.

Pattberg, Philipp. 2005. What role for private rule-making in global environmental governance? Analysing the Forest Stewardship Council (FSC). International Environmental Agreements: Politics, Law and Economics 5(2):175-189.

Pattberg, Philipp. 2007. Private institutions and global governance: the new politics of environmental sustainability. Cheltenham: Edward Elgar.

Pattberg, Philipp. 2012. The role and relevance of networked climate governance. In Global climate governance beyond 2012: architecture, agency and adaptation, Hrsg. Frank Biermann, Philipp Pattberg, und Fariborz Zelli, 146-164. Cambridge: Cambridge University Press. 
Pingeot, Lou. 2014. Contracting insecurity. Private military and security companies and the future of the United Nations. Bonn/New York: Global Policy Forum/Rosa-Luxemburg-Stiftung.

Ponte, Stefano. 2014. "Roundtabling" sustainability: lessons from the biofuel industry. Geoforum 54:261-271.

Potoski, Matthew, und Aseem Prakash. 2005. Green clubs and voluntary governance: ISO 14001 and firms' regulatory compliance. American Journal of Political Science 49(2):235-248.

Prem, Berenike. 2020. Private military and security companies as global governors: from barricades to boardrooms. Abingdon, New York: Routledge.

Prem, Berenike. 2021. The regulation of private military and security companies: analyzing power in multistakeholder initiatives. Contemporary Security Policy 42(3):345-370.

Price, Richard. 2003. Transnational civil society and advocacy in world politics. World Politics 55(4): 579-606.

Rasche, Andreas. 2009. A necessary supplement—what the United Nations global compact is and is not. Business and Society 48(4):511-537.

Raustiala, Kal. 1997. States, NGOs and international environmental institutions. International Studies Quartely 41(4):719-740.

Reimann, Kim D. 2006. A view from the top: international politics, norms and the worldwide growth of NGOs. International Studies Quarterly 50(1):45-68.

Risse-Kappen, Thomas. 1995. Bringing transnational relations back in: non-state actors, domestic structures, and international institutions. Cambridge: Cambridge University Press.

Ruggie, John G. 2013. Just business-multinational corporations and human rights. New York: Norton.

Scherler, Patrik. 1996. Management der Krisenkommunikation: Theorie und Praxis zum Fall Brent Spar (Greenpeace gegen Royal Dutch/Shell). Basel: Helbing \& Lichtenhahn.

Schwartz, Moshe. 2011. The department of defense's use of private security contractors in Afghanistan and Iraq: background, analysis, and options for congress. February 21. Report for Congress. Washington D.C.: Congressional Research Service.

Sethi, Prakash S., und Janet L. Rovenpor. 2016. The role of NGOs in ameliorating sweatshop-like conditions in the global supply chain: the case of fair labor association (FLA) and social accountability international (SAI). Business and Society Review 121(1):5-36.

Shawki, Noha. 2010. Issue frames and the political outcomes of transnational campaigns: a comparison of the jubilee 2000 movement and the currency transaction tax campaign. Global Society 24(2):203-230.

Shift. 2021. Business learning program. https://shiftproject.org/what-we-do/business/business-learning/. Zugegriffen: 23. März 2021.

Staggenborg, Suzanne. 2013. Institutionalization of social movements. In The Wiley-Blackwell encyclopedia of social and political movements. : Blackwell.

Steffek, Jens. 2013. Explaining cooperation between IGOs and NGOs—push factors, pull factors, and the policy cycle. Review of International Studies 39(04):993-1013.

Stürchler, Nikolas. 2008. The Swiss initiative comes alive. Seventeen states agree on how to legally deal with private security companies. Journal of International Peace Operations 4(3):9-12.

Tallberg, Jonas. 2010. Transnational access to international institutions: three approaches. In Transnational actors in global governance: patterns, explanations and implications, Hrsg. Christer Jönsson, Jonas Tallberg, 45-66. London: Palgrave Macmillan.

Tosun, Jale, Sebastian Koos, und Jennifer Shore. 2016. Co-governing common goods: interaction patterns of private and public actors. Policy and Society 35(1):1-12.

Treaty Alliance. 2013. 2013 Bangkok joint statement. https://www.treatymovement.com/statement-2013. Zugegriffen: 15. März 2021.

United Nations. 2016. The power of principles: the ten principles of the UN global compact. https://www. unglobalcompact.org/what-is-gc/mission/principles. Zugegriffen: 10. Dez. 2016.

Utting, Peter. 2000. Business responsibility for sustainable development. Occasional paper no. 2. Geneva: UNRISD.

Utting, Peter. 2005. Corporate responsibility and the movement of business. Development in Practice 15(3/4):375-388.

Voillat, Claude. 2012. Pushing the humanitarian agenda through engagement with business actors: the ICRC's experience. International Review of the Red Cross 94(887):1089-1114.

War on Want. 2008. G4S workers backed in living wage. https://web.archive.org/web/20100616112702/ http://www.waronwant.org/news/247-media/15492-g4s-workers-backed-on-living-wage. Zugegriffen: 24. März 2021. 
War on Want. 2012. G4S olympics games security row sparks call for mercenary regulation. https:// web.archive.org/web/20130308044832/https:/www.waronwant.org/news/press-releases/17605-g4sgames-security-row-sparks-call-for-regulation. Zugegriffen: 24. März 2021.

War on Want. 2013. G4S accused over links to palestinian 'torture' death jail. http://www.waronwant.org/ news/press-releases/17819-g4s-accused-over-links-to-palestinian-torture-death-jail. Zugegriffen: 1. Juli 2013.

War on Want. 2014. Gates foundation sells down its shares in G4S. http://www.waronwant.org/media/ gates-foundation-sells-down-its-shares-g4s. Zugegriffen: 17. Juli 2015.

Williams, Oliver F. 2004. The UN global compact: the challenge and the promise. Business Ethics Quarterly 14(4):755-774.

Witte, Jan Martin, Wolfgang H. Reinicke, und Thorsten Benner. 2000. Beyond multilateralism: global public policy networks. Internationale Politik und Gesellschaft 2000:2176-2188.

World Wide Fund for Nature. 2020. Zusammenarbeit mit Unternehmen. https://www.wwf.de/ zusammenarbeit-mit-unternehmen/. Zugegriffen: 18. Juni 2020.

Yamin, Farhana. 2001. NGOs and international environmental law: a critical evaluation of their roles and responsibilities. Review of European Comparative \& International Environmental Law 10(2):149-162.

Yaziji, Michael, und Jonathan P. Doh. 2009. NGOs and corporations: conflict and collaboration. Business, value creation, and society. Cambridge: Cambridge University Press.

Zangl, Bernhard, und Michael Zürn. 2003. Frieden und Krieg: Sicherheit in der nationalen und postnationalen Konstellation. Frankfurt: Suhrkamp. 